\title{
Computing Extensions of Linear Codes
}

\author{
Markus Grassl \\ Institut für Algorithmen und Kognitive Systeme \\ Fakultät für Informatik, Universität Karlsruhe (TH) \\ Am Fasanengarten 5, 76128 Karlsruhe, Germany \\ Email: grassl@ira.uka.de
}

\begin{abstract}
This paper deals with the problem of increasing the minimum distance of a linear code by adding one or more columns to the generator matrix. Several methods to compute extensions of linear codes are presented. Many codes improving the previously known lower bounds on the minimum distance have been found.
\end{abstract}

\section{INTRODUCTION}

In this paper we consider the question when a linear code $C=[n, k, d]_{q}$ over $\mathbb{F}_{q}$ of length $n$, dimension $k$, and minimum distance $d$ can be extended to a code $C^{\prime}=[n+1, k, d+1]_{q}$. It is a well known fact in coding theory that every binary linear code $C=[n, k, d]_{2}$ whose minimum weight $d$ is odd can be extended to a code $[n+1, k, d+1]_{2}$ by adding a single parity check. This can also be expressed in terms of Construction $\mathrm{X}$ [17] applied to the code $C$, its one-codimensional evenweight subcode $C_{0}$, and the trivial code $[1,1,1]_{2}$. While this result does not have an immediate generalization to nonbinary alphabets, Hill and Lizak [9], [10] proved the following theorem:

Theorem 1: Let $C$ be an $[n, k, d]_{q}$ code with $\operatorname{gcd}(d, q)=1$ and with all weights congruent to 0 or $d$ (modulo $q$ ). Then $C$ can be extended to an $[n+1, k, d+1]_{q}$ code all of whose weights are congruent to 0 or $d+1$ (modulo $q$ ).

In order to apply this theorem, knowledge about the weight spectrum of the code $C$ is required. A generalization of this theorem due to Simonis [16] can be applied when additionally information on the weight distribution of the code $C$ is available. The special cases with $\operatorname{gcd}(q, d)=1$ and in particular ternary codes have been treated by Maruta [13][15]. However, these results are of rather theoretical nature and have mainly be used to prove the non-existence of codes with certain parameters. The application to a specific code might be difficult since one has to compute information on the weight distribution of the code first.

\section{Extension Based on Minimum Weight CODEWORDS}

\section{A. The main criterion}

In the following, we consider the problem to test if a code $C=[n, k, d]_{q}$ which is explicitly given by a generator matrix $G$ can be extended and to compute an extension if it exists. Based on the set of all codewords of minimum weight, we get the following criterion for the extendability of a linear code:

Theorem 2: Let $C=[n, k, d]_{q}$ be a linear code over $\mathbb{F}_{q}$ with minimum distance $d$. Furthermore, let $G \in \mathbb{F}_{q}^{k \times n}$ be a generator matrix for $C$ of full rank. By $\mathcal{S}_{d}=\{\boldsymbol{c} \in C \mid \operatorname{wgt} \boldsymbol{c}=$ $d\}$ we denote the set of all codewords of minimum weight and by $\mathcal{J}_{d}=\left\{\boldsymbol{v} \in \mathbb{F}_{q}^{k} \mid \operatorname{wgt}(\boldsymbol{v} G)=d\right\}$ we denote the corresponding information vectors.

The code $C$ can be extended to a code $C^{\prime}=[n+m, k, d+$ $1]_{q}$ if and only if there is a matrix $X \in \mathbb{F}_{q}^{k \times m}$ such that

$$
\sum_{i=1}^{k} v_{i} X_{i} \neq 0 \quad \text { for all } \boldsymbol{v} \in \mathcal{J}_{d},
$$

where $X_{i}$ denotes the $i$-th row of the matrix $X$.

Proof: Let $G^{\prime}=(G \mid X)$ be the matrix that is obtained by appending the matrix $X$ to $G$. Encoding an information vector $\boldsymbol{v}$ with the matrix $G^{\prime}$ we get

$$
\boldsymbol{c}^{\prime}=\boldsymbol{v} G^{\prime}=\left(\boldsymbol{v} G \mid \sum_{i=1}^{k} v_{i} X_{i}\right)
$$

The weight of a non-zero codeword $\boldsymbol{c}^{\prime}$ is $d$ if and only if $\operatorname{wgt}(\boldsymbol{v} G)=d$ and $\sum_{i=1}^{k} v_{i} X_{i}=0$.

In particular we consider the extension by a single column:

Corollary 3: Using the notation of Theorem 2, a linear code $C=[n, k, d]_{q}$ can be extended to a code $C^{\prime}=[n+1, k, d+1]_{q}$ if and only if there exists a column vector $\boldsymbol{x} \in \mathbb{F}_{q}^{k}$ such that

$$
\sum_{i=1}^{k} v_{i} x_{i} \neq 0 \text { for all } \boldsymbol{v} \in \mathcal{J}_{d}
$$

In order to apply criterion (1) or (2), we have to compute the set $\mathcal{J}_{d}$ of information vectors of all codewords of minimum weight.

\section{B. Computing the minimum weight codewords}

In the sequel we describe an algorithm to compute the minimum distance of a code as well as all words of minimum weight. The algorithm is based on an algorithm by Zimmermann to compute the minimum distance (see [19] and [1, Algorithmus 1.3.6]) which improved an algorithm by Brouwer. Together with some further improvements, the algorithm is implemented in the computer algebra system MAGMA (see [2], [8]).

The main idea of the algorithm is to enumerate the codewords in such a way that one does not only obtain an upper bound on the minimum distance of the code via the minimum of the weight of the words that have been encountered, but to establish lower bounds on the minimum distance as well. For this, we are using a collection of systematic generator matrices 
$G_{j}$ with corresponding information sets $\mathcal{I}_{j}$. Given an ordered list $\left(\mathcal{I}_{1}, \mathcal{I}_{2}, \ldots, \mathcal{I}_{\mu}\right)$ of information sets, we define the relative rank $r_{j}$ of $\mathcal{I}_{j}$ as

$$
r_{j}:=k-\left|\mathcal{I}_{j} \cap \bigcup_{l=1}^{j-1} \mathcal{I}_{l}\right|,
$$

i.e., $r_{j}$ equals the number of positions in the information set $\mathcal{I}_{j}$ that are disjoint from all informations sets $\mathcal{I}_{l}$ with $l<j$. If we now encode all words $i \in \mathbb{F}_{q}^{k}$ of weight wgt $i \leq w$ using all systematic generator matrices, we know that the weight of the remaining codewords is at least

$$
d_{\mathrm{lb}}:=\sum_{j=1}^{\mu} \max \left(0,(w+1)-\left(k-r_{j}\right)\right),
$$

as the weight in each corresponding information set is at least $w+1$, and we have to subtract the positions which have been double-counted due to overlapping information sets.

Algorithm 4 (Minimum Weight Words):

MinimumWeightWords:=function (C);

minwords: $=\emptyset$;

$d_{1 \mathrm{~b}}:=1$;

$d_{\mathrm{ub}}:=n-k+1$

$w:=1$;

while $w \leq k$ and $d_{1 \mathrm{~b}} \leq d_{\mathrm{ub}}$ do

for $j:=1$ to $\mu$ do

words : $=\left\{\boldsymbol{i} \cdot G_{j}: \boldsymbol{i} \in \mathbb{F}_{q}^{k} \mid \operatorname{wgt}(\boldsymbol{i})=w\right\} ;$

$\mathrm{d}:=\min \{\operatorname{wgt}(\boldsymbol{c}): \boldsymbol{c} \in$ words $\}$;

if $d<d_{\text {ub }}$ then

$d_{\mathrm{ub}}:=\mathrm{d}$;

minwords $:=\left\{\boldsymbol{c}: \boldsymbol{c} \in\right.$ words $\left.\mid \operatorname{wgt}(\boldsymbol{c})=d_{\mathrm{ub}}\right\} ;$ else

minwords join $:=\left\{\boldsymbol{c}: \boldsymbol{c} \in \text { words } \mid \operatorname{wgt}(\boldsymbol{c})=d_{\mathrm{ub}}\right\}_{i}$ end if;

end for;

$d_{1 \mathrm{~b}}:=\sum_{j=1}^{\mu} \max \left(0,(w+1)-\left(k-r_{j}\right)\right)$;

$w:=w+1$;

end while;

return minwords;

end function;

With a slight modification, this algorithm can also be used to compute all codewords of a given weight or all codewords whose weight is below a certain value. The total number of encodings to find all codewords of weight not exceeding $d$ is given by

$$
\sum_{w=1}^{w_{0}} \mu\left(\begin{array}{l}
k \\
w
\end{array}\right)(q-1)^{w-1}
$$

where $w_{0}$ is the minimum value such that

$$
\sum_{j=1}^{\mu} \max \left(0,\left(w_{0}+1\right)-\left(k-r_{j}\right)\right)>d .
$$

Of course, if (3) is larger than $q^{k}$, one should directly enumerate all codewords instead of using Algorithm 4 . But in most cases, using more than one generator matrix results in an overall saving as the maximum weight $w_{0}$ of the vectors $\boldsymbol{i}$ that has to be considered is smaller, and (3) grows only linear in $\mu$, but exponential in $w_{0}$. If partial knowledge of the automorphism group of the code is available, which is e. g. the case for cyclic or quasi-cyclic codes, the lower bound (4) on $w_{o}$ can be improved so that the overall complexity for computing the minimum weight codewords is reduced [4], [8], [18].

\section{COMPUting EXTENSIONS}

\section{A. Exhaustive search}

Given the set $\mathcal{J}_{d}$ of information vectors of the minimum weight codewords, one can use an exhaustive search to find a column vector $x$ or a matrix $X$ that fulfills condition (2) or (1). In total there are $q^{m k}-1$ non-zero matrices. As both conditions are bilinear, it suffices to consider normalized information vectors and may normalize the columns in the matrix $X$, reducing the total number of matrices by no more than the factor $(q-1)^{m}$. Sorting the columns of the matrix gives an additional reduction by a factor of at most $m$ !. Hence using this approach, one has to test at least

$$
\frac{q^{m k}-1}{m !(q-1)^{m}}
$$

matrices in order to show that no extension exists. If one is interested in all possible extension, an exhaustive search is necessary, too. Nonetheless, exhaustive search might be feasible to find an extension if the dimension $k$ of the code is small or if many extensions exist.

\section{B. Extending binary codes by one position}

For binary codes, condition (2) can be re-written as

$$
\sum_{i=1}^{k} v_{i} x_{i}=1 \quad \text { for all } \boldsymbol{v} \in \mathcal{J}_{d} .
$$

The possible extensions of the code correspond to the set of solutions of the inhomogeneous system of linear equations (6). The complexity of computing the solutions if one exists is no longer exponential as in (5), but only polynomial. Moreover, it suffices to compute a subset $\mathcal{J}_{d}^{\prime}$ of the information vectors of the minimum weight codewords such that the linear spans of $\mathcal{J}_{d}$ and $\mathcal{J}_{d}^{\prime}$ coincide.

\section{Extensions by one via solving polynomial equations}

For non-binary codes, condition (2) does not directly translate into an equation. However, using the fact that the roots of the polynomial $y^{q-1}-1 \in \mathbb{F}_{q}[y]$ are exactly the non-zero elements of $\mathbb{F}_{q}$, we get the condition

$$
\left(\sum_{i=1}^{k} v_{i} x_{i}\right)^{q-1}=1 \quad \text { for all } \boldsymbol{v} \in \mathcal{J}_{d} .
$$

The set of all solutions of conditions (7) is characterized by the ideal

$$
J:=\left\langle\left(\sum_{i=1}^{k} v_{i} x_{i}\right)^{q-1}-1: \boldsymbol{v} \in \mathcal{J}_{d}\right\rangle \unlhd \mathbb{F}_{q}\left[x_{1}, \ldots, x_{k}\right]
$$

in the polynomial ring $\mathbb{F}_{q}\left[x_{1}, \ldots, x_{k}\right]$ in $k$ variables over $\mathbb{F}_{q}$. Testing whether the system of polynomial equations (7) has a 
solution and computing the solutions can be done e.g. using Gröbner bases [5]. The system does not have a solution if and only if a Gröbner basis of the ideal $J$ contains a nonzero constant polynomial. In general, it is difficult to estimate the complexity of computing a particular Gröbner basis, and the complexity might be exponential. However, computing a Gröbner basis without homogenization quite often quickly shows that there is no solution. Using the algorithm $F_{4}$ of Faugére to compute a Gröbner basis [6] as implemented in the computer algebra system MAGMA [2], it was quite often faster to compute all solutions via the Gröbner basis than finding a single solution using exhaustive search (see below).

\section{General extensions via solving polynomial equations}

For both binary and non-binary codes, condition (1) can be expressed in terms of polynomial equations. A vector $\boldsymbol{y} \in \mathbb{F}_{q}^{m}$ is non-zero if and only if at least one coordinate is non-zero, i.e.

$$
\prod_{j=1}^{m}\left(y_{j}^{q-1}-1\right)=0 .
$$

Hence the solutions of (1) are characterized by the ideal

$$
J:=\left\langle\prod_{j=1}^{m}\left(\left(\sum_{i=1}^{k} v_{i} X_{i j}\right)^{q-1}-1\right): \boldsymbol{v} \in \mathcal{J}_{d}\right\rangle
$$

in the polynomial ring $\mathbb{F}_{q}\left[X_{11}, \ldots, X_{k m}\right]$ in $k m$ variables over $\mathbb{F}_{q}$. Note that even for $q=2$, the conditions are no longer linear, but of degree $m$.

\section{E. Further remarks}

For linear binary codes we have seen that sometimes it is sufficient to compute only a subset of the minimum weight codewords. In general, one can use a subset of $\mathcal{J}_{d}$ to test whether a code can be extended and compute a set of candidates for the extension using the ideal $J$ of eq. (8) or eq. (9). In many cases, the resulting set of candidates is rather small, so that one can perform an exhaustive search among them. Similar, a double extension of a code $C$ to a code $C^{\prime \prime}=[n+2, k, d+2]_{q}$ can be found using the solutions for the single extension to $C^{\prime}=[n+1, k, d+1]_{q}$.

Kohnert [11], [12] has proposed to compute extensions using integer linear programming by reformulating (1) as hittingset problem. The ground set of the hitting-set problem is the set of all normalized non-zero vectors that can be appended to the generator matrix, so its size grows exponentially in the dimension of the code.

\section{EXAMPLES}

We tested the various methods using the best known linear codes (BKLC) from MAGMA and the linear codes from [7] which establish or improve the lower bound on the minimum distance in Brouwer's tables [3]. We have not found any binary code that can be extended by one position, but many codes over $\mathbb{F}_{q}$ for $q=3,4,5,7,8,9$. In Table $\square$ we list 71 of these codes together with some timing information. The columns with headings $\mathcal{S}_{d}$ and $\left|\mathcal{S}_{d}\right|$ provide the time to compute all minimum weight words and the number of minimum weight words. In the columns full iteration and iteration the time needed to find all or just one solution by exhaustive search (see Sect. [II-A) is given for some of the codes. The next four columns provide information on the approach of Sect. III-C solving a system of polynomial equations. We have used the additional equations $x_{1}^{2}-x_{1}$ which ensures that the first component of the column vector $\boldsymbol{x}$ is either zero or one, and $x_{j}^{q}-x_{j}$ for $j=2, \ldots, k$ as all entries of $\boldsymbol{x}$ are elements of $\mathbb{F}_{q}$. The total running time is dominated by the time needed to compute the Gröbner basis, the construction of the equations and computing the solutions can be neglected in most of the cases. In the final column the total number of solutions is given, where we have identified solutions that differ by a nonzero scalar factor.

With some few exceptions, e. g., for the codes $[89,11,54]_{5}$, $[93,11,57]_{5},[76,8,53]_{7},[45,8,30]_{9}$, computing all solutions via a Gröbner basis is even faster than finding a single solution by exhaustive search.

Table [II contains some binary and ternary codes whose minimum distance can be increased by appending two columns to the generator matrix. For these codes, the Gröbner basis approach is quite fast, but unfortunately, this is not always true.

There is a ternary code $C=[178,23,81]_{3}$ with 80 words of weight 81 that can be extended to a code $C^{\prime}=[179,23,82]_{3}$. Computing a Gröbner basis took about 79 hours on an AMD Opteron 252 (clock speed $2.6 \mathrm{GHz}$ ), using about $16 \mathrm{~GB}$ of memory. Using exhaustive search, a solution was found in 189.730 seconds, while the projected total running time for the complete exhaustive search is more than 250 hours.

Furthermore, there is a quasicyclic code $C=[140,19,73]_{4}$ with 840 words of weight 73 that can be extended to a code $C^{\prime}=[142,19,74]$. Using exhaustive search, a solution was found after 4.36 hours on an AMD Opteron 250 (clock speed $2.4 \mathrm{GHz}$ ). After 35.75 days of CPU time, 654 solutions have been found while the projected total running time for the exhaustive search is $10^{11}$ years. Computing a Gröbner basis for the ideal $J$ of this code seems to be infeasible.

It turns out that the codes with parameters $[66,22,22]_{3}$, $[67,23,22]_{3}, \quad[78,11,47]_{5}, \quad[51,6,37]_{7}$, and $[76,8,53]_{7}$ are doubly extendible. The codes $[172,17,70]_{2}$ and $[173,18,70]_{2}$ in Table $\Pi$ can be extended in two steps to codes $[175,17,72]_{2}$ and $[176,18,72]_{2}$. The codes $[119,7,75]_{3}$ and $[85,9,51]_{3}$ can even be extended to codes $[123,7,78]_{3}$ and $[89,9,54]_{3}$.

\section{ACKNOWLEDGMENT}

The author would like to thank John Cannon, Allan Steel, and Greg White from the MAGMA group, University of Sydney, for their support.

\section{REFERENCES}

[1] A. Betten, H. Fripertinger, A. Kerber, A. Wassermann, and K.-H Zimmermann, Codierungstheorie: Konstruktionen und Anwendungen linearer Codes. Berlin: Springer, 1998.

[2] W. Bosma, J. J. Cannon, and C. Playoust, "The Magma Algebra System I: The User Language," Journal of Symbolic Computation, vol. 24, no. 3-4, pp. 235-265, 1997. 
TABLE I

CODES $C=[n, k, d]_{q}$ THAT CAN BE EXTEND TO CODES $C^{\prime}=[n+1, k, d+1]_{q}$.

\begin{tabular}{|c|c|c|c|c|c|c|c|c|c|}
\hline code & computing $\mathcal{S}_{d}$ & $\left|\mathcal{S}_{d}\right|$ & full iteration & iteration & equations & Gröbner & solution & total Gröbner & \#solutions \\
\hline$[233,9,146]_{3}$ & 0.130 & 1410 & 0.150 & 0.020 & 0.020 & 0.040 & 0.000 & 0.080 & 1 \\
\hline$[86,10,49]_{3}$ & 0.020 & 1008 & 0.430 & 0.030 & 0.020 & 0.030 & 0.000 & 0.050 & 3 \\
\hline$[175,10,103]_{3}$ & 0.100 & 352 & 0.440 & 0.010 & 0.010 & 0.010 & 0.000 & 0.020 & 1 \\
\hline$[87,11,49]_{3}$ & 0.030 & 3312 & 1.250 & 0.280 & 0.070 & 0.100 & 0.000 & 0.180 & 2 \\
\hline$[176,13,97]_{3}$ & 0.120 & 66 & 11.370 & 0.000 & 0.000 & 0.140 & 0.000 & 0.150 & 9 \\
\hline$[100,19,43]_{3}$ & 2.080 & 21140 & 10602.910 & 1920.910 & 0.980 & 4.260 & 0.000 & 5.310 & 1 \\
\hline$[102,19,44]_{3}$ & 2.540 & 14492 & 9893.640 & 1257.940 & 0.650 & 2.020 & 0.000 & 2.710 & 1 \\
\hline$[166,19,81]_{3}$ & 11.640 & 328 & 9686.610 & 189.260 & 0.020 & 0.130 & 0.010 & 0.160 & 9 \\
\hline$[104,20,44]_{3}$ & 4.010 & 15722 & - & 3513.530 & 0.770 & 2.420 & 0.000 & 3.250 & 1 \\
\hline$[66,22,22]_{3}$ & 0.690 & 90 & - & 160.600 & 0.000 & 780.780 & 26.570 & 807.380 & 465 \\
\hline$[108,22,43]_{3}$ & 13.450 & 102 & - & 1820.900 & 0.000 & 604.690 & 0.010 & 604.710 & 12 \\
\hline$[165,22,75]_{3}$ & 140.230 & 96 & - & 116.360 & 0.010 & 793.850 & 0.260 & 794.130 & 92 \\
\hline$[67,23,22]_{3}$ & 0.440 & 134 & - & 8931.530 & 0.010 & 43.320 & 2.450 & 45.800 & 201 \\
\hline$[97,23,37]_{3}$ & 24.150 & 746 & - & 154487.840 & 0.040 & 0.130 & 0.000 & 0.170 & 1 \\
\hline$[99,23,38]_{3}$ & 30.600 & 658 & - & 160156.420 & 0.030 & 0.100 & 0.000 & 0.140 & 1 \\
\hline$[111,23,44]_{3}$ & 40.170 & 114 & - & 119090.890 & 0.000 & 905.710 & 0.000 & 905.720 & 3 \\
\hline$[149,23,64]_{3}$ & 149.090 & 108 & - & 11522.410 & 0.010 & 1147.910 & 0.010 & 1147.930 & 23 \\
\hline$[166,23,75]_{3}$ & 238.290 & 200 & - & 35682.570 & 0.010 & 10.390 & 0.000 & 10.400 & 3 \\
\hline$[191,23,89]_{3}$ & 420.910 & 98 & - & 1736.210 & 0.000 & 9430.260 & 0.690 & 9430.960 & 123 \\
\hline$[191,24,88]_{3}$ & 722.510 & 112 & - & 3220.530 & 0.010 & 2265.550 & 0.010 & 2265.570 & 15 \\
\hline$[194,24,90]_{3}$ & 830.840 & 112 & - & 32986.960 & 0.010 & 2262.690 & 0.000 & 2262.700 & 13 \\
\hline$[197,24,92]_{3}$ & 1050.770 & 110 & - & 10215.69 & 0.010 & 2396.040 & 0.030 & 2396.090 & 32 \\
\hline$[194,25,89]_{3}$ & 2390.880 & 114 & - & 5909.010 & 0.000 & 6391.800 & 0.060 & 6391.860 & 39 \\
\hline$[215,25,103]_{3}$ & 5443.010 & 164 & - & 150817.640 & 0.010 & 97.980 & 0.000 & 97.990 & 1 \\
\hline$[178,27,77]_{3}$ & 13427.570 & 126 & - & 301975.480 & 0.010 & 127409.310 & 0.070 & 127409.400 & 41 \\
\hline$[127,28,49]_{3}$ & 7995.850 & 12440 & - & - & 0.920 & 1.880 & 0.000 & 2.860 & 4 \\
\hline$[135,6,96]_{4}$ & 0.030 & 225 & 0.050 & 0.000 & 0.010 & 0.000 & 0.000 & 0.010 & 2 \\
\hline$[159,7,111]_{4}$ & 0.050 & 2604 & 0.180 & 0.020 & 0.030 & 0.070 & 0.000 & 0.130 & 1 \\
\hline$[241,7,174]_{4}$ & 0.130 & 804 & 0.170 & 0.010 & 0.010 & 0.020 & 0.000 & 0.030 & 1 \\
\hline$[190,8,130]_{4}$ & 0.200 & 4164 & 0.720 & 0.030 & 0.080 & 0.080 & 0.000 & 0.170 & 3 \\
\hline$[191,8,130]_{4}$ & 0.130 & 4158 & 0.720 & 0.030 & 0.080 & 0.080 & 0.000 & 0.170 & 4 \\
\hline$[132,11,81]_{4}$ & 0.160 & 777 & 43.430 & 1.140 & 0.020 & 0.040 & 0.000 & 0.070 & 1 \\
\hline$[94,13,53]_{4}$ & 0.420 & 16890 & 738.800 & 1.760 & 0.540 & 1.270 & 0.000 & 1.870 & 3 \\
\hline$[129,13,77]_{4}$ & 0.660 & 15312 & 865.230 & 221.230 & 0.540 & 1.060 & 0.000 & 1.670 & 2 \\
\hline$[132,13,79]_{4}$ & 0.680 & 17136 & 747.580 & 217.610 & 0.630 & 1.280 & 0.000 & 1.980 & 3 \\
\hline$[142,13,85]_{4}$ & 0.720 & 8049 & 737.340 & 33.000 & 0.270 & 0.500 & 0.000 & 0.810 & 1 \\
\hline$[149,13,90]_{4}$ & 1.140 & 18318 & 764.780 & 21.600 & 0.660 & 2.020 & 0.010 & 2.750 & 4 \\
\hline$[161,13,98]_{4}$ & 1.340 & 31884 & 817.810 & 229.780 & 1.280 & 4.870 & 0.000 & 6.300 & 2 \\
\hline$[196,13,122]_{4}$ & 1.380 & 168 & 745.220 & 14.860 & 0.000 & 43.960 & 0.000 & 43.970 & 3 \\
\hline$[120,14,69]_{4}$ & 1.920 & 315 & 2989.640 & 0.010 & 0.010 & 0.880 & 13.190 & 14.150 & 729 \\
\hline$[182,14,110]_{4}$ & 3.530 & 19698 & 3142.570 & 741.160 & 0.760 & 2.430 & 0.000 & 3.280 & 6 \\
\hline$[134,15,77]_{4}$ & 5.220 & 50793 & 12051.040 & 463.570 & 2.110 & 13.010 & 0.000 & 15.350 & 4 \\
\hline$[183,15,110]_{4}$ & 13.190 & 49218 & 12193.490 & 3525.470 & 2.320 & 18.940 & 0.010 & 21.500 & 3 \\
\hline$[45,16,17]_{4}$ & 0.180 & 192 & 47480.220 & 2358.470 & 0.010 & 2833.000 & 0.000 & 2833.010 & 3 \\
\hline$[91,16,47]_{4}$ & 6.430 & 3330 & - & 1831.150 & 0.120 & 0.180 & 0.000 & 0.300 & 1 \\
\hline$[136,16,75]_{4}$ & 18.300 & 38880 & - & 308.820 & 1.580 & 6.940 & 0.000 & 8.700 & 18 \\
\hline$[176,16,103]_{4}$ & 29.980 & 219 & - & 2779.760 & 0.010 & 3747.880 & 0.000 & 3747.890 & 1 \\
\hline$[64,17,29]_{4}$ & 4.430 & 6048 & - & 699.140 & 0.220 & 0.340 & 0.000 & 0.580 & 3 \\
\hline$[116,17,61]_{4}$ & 25.710 & 249 & - & 1.660 & 0.010 & 8275.260 & 0.320 & 8275.610 & 243 \\
\hline$[137,17,75]_{4}$ & 37.240 & 122751 & - & 2731.850 & 5.870 & 107.510 & 0.000 & 113.990 & 3 \\
\hline$[172,17,99]_{4}$ & 83.180 & 65325 & - & 1379.040 & 3.230 & 30.070 & 0.000 & 33.670 & 27 \\
\hline$[87,19,41]_{4}$ & 125.400 & 2550 & - & 126.980 & 0.130 & 0.520 & 0.000 & 0.660 & 4 \\
\hline$[95,19,45]_{4}$ & 50.430 & 11451 & - & 43493.400 & 0.590 & 1.230 & 0.000 & 1.880 & 1 \\
\hline$[110,19,54]_{4}$ & 31.710 & 330 & - & 177473.000 & 0.010 & 28449.570 & 0.000 & 28449.590 & 5 \\
\hline
\end{tabular}

Timings in seconds using Magma V2.13-8 on an AMD Opteron 252 (clock speed 2.6 GHz, 16 GB RAM); for $q=3$, an AMD Opteron 254 (clock speed $2.8 \mathrm{GHz}, 16 \mathrm{~GB}$ RAM) has been used. 
TABLE【 (continued)

Codes $C=[n, k, d]_{q}$ That CAN Be EXTEND to Codes $C^{\prime}=[n+1, k, d+1]_{q}$.

\begin{tabular}{c|r|r|r|r|rrrr|r} 
code & computing $\mathcal{S}_{d}$ & $\left|\mathcal{S}_{d}\right|$ & full iteration & iteration & equations & Gröbner & solution & total Gröbner & \#solutions \\
\hline$[105,7,77]_{5}$ & 0.040 & 1760 & 1.010 & 0.070 & 0.050 & 0.230 & 0.000 & 0.280 & 1 \\
{$[78,11,47]_{5}$} & 0.060 & 780 & 682.690 & 53.860 & 0.060 & 55.730 & 0.000 & 55.790 & 3 \\
{$[84,11,51]_{5}$} & 0.220 & 3424 & 667.630 & 0.020 & 0.300 & 1.900 & 0.000 & 2.220 & 3 \\
{$[89,11,54]_{5}$} & 0.180 & 232 & 683.130 & 3.170 & 0.020 & 4324.910 & 0.020 & 4324.950 & 35 \\
{$[93,11,57]_{5}$} & 0.270 & 224 & 659.400 & 0.630 & 0.020 & 4172.490 & 0.060 & 4172.570 & 45 \\
\hline$[65,4,53]_{7}$ & 0.020 & 408 & 0.040 & 0.000 & 0.010 & 0.010 & 0.000 & 0.020 & 1 \\
{$[51,6,37]_{7}$} & 0.020 & 504 & 2.070 & 0.000 & 0.030 & 0.360 & 0.000 & 0.390 & 14 \\
{$[76,8,53]_{7}$} & 0.030 & 912 & 102.710 & 1.080 & 0.080 & 249.500 & 0.000 & 249.590 & 4 \\
\hline$[44,8,29]_{8}$ & 0.020 & 2443 & 376.920 & 2.830 & 0.090 & 2.240 & 0.000 & 2.340 & 1 \\
{$[68,8,49]_{8}$} & 0.100 & 12936 & 338.610 & 11.270 & 0.670 & 0.830 & 0.000 & 1.550 & 1 \\
{$[27,9,15]_{8}$} & 0.020 & 4914 & 2760.190 & 52.360 & 0.170 & 2.140 & 0.000 & 2.330 & 1 \\
{$[69,9,49]_{8}$} & 0.300 & 25480 & 2804.850 & 19.510 & 1.870 & 2.810 & 0.000 & 4.800 & 1 \\
\hline$[82,5,67]_{9}$ & 0.150 & 2176 & 1.700 & 0.030 & 0.050 & 0.200 & 0.000 & 0.260 & 1 \\
{$[87,6,69]_{9}$} & 0.400 & 4256 & 15.750 & 0.050 & 0.140 & 0.800 & 0.010 & 0.970 & 3 \\
{$[127,6,103]_{9}$} & 0.230 & 976 & 15.310 & 0.500 & 0.030 & 4.170 & 0.000 & 4.210 & 1 \\
{$[98,7,76]_{9}$} & 0.860 & 6776 & 146.120 & 1.430 & 0.360 & 2.550 & 0.000 & 2.950 & 1 \\
{$[45,8,30]_{9}$} & 0.220 & 1408 & 1332.960 & 325.270 & 0.070 & 10637.000 & 0.000 & 10637.080 & 1
\end{tabular}

Timings in seconds using Magma V2.13-8 on an AMD Opteron 252 (clock speed 2.6 GHz, 16 GB RAM).

TABLE II

CODES $C=[n, k, d]_{q}$ THAT CAN BE EXTEND TO CODES $C^{\prime}=[n+2, k, d+1]_{q}$.

\begin{tabular}{c|r|r|r|r|r}
$C=[n, k, d]_{q}$ & $\mathcal{S}_{d}$ & $\left|\mathcal{S}_{d}\right|$ & iteration & Gröbner & \#solutions \\
\hline$[205,13,94]_{2}$ & 0.070 & 2169 & 152.280 & 0.440 & 3 \\
{$[172,17,70]_{2}$} & 0.060 & 2616 & 71392.360 & 0.860 & 3 \\
{$[166,18,66]_{2}$} & 0.050 & 1800 & - & 0.600 & 3 \\
{$[173,18,70]_{2}$} & 0.080 & 4230 & - & 1.570 & 3 \\
{$[205,19,82]_{2}$} & 0.080 & 1632 & - & 0.600 & 9 \\
\hline$[119,7,75]_{3}$ & 0.020 & 756 & 16.510 & 0.710 & 6 \\
{$[85,9,51]_{3}$} & 0.030 & 4536 & 143.400 & 3.500 & 24
\end{tabular}

Timings in seconds using Magma V2.13-8 on an AMD Opteron 254, clock speed $2.8 \mathrm{GHz}, 16 \mathrm{~GB}$ RAM.

[3] A. E. Brouwer, "Bounds on the Size of Linear Codes," in Handbook of Coding Theory, V. S. Pless and W. C. Huffman, Eds. Amsterdam: Elsevier, 1998, pp. 295-461.

[4] C.-L. Chen, "Computer Results on the Minimum Distance of Some Binary Cyclic Codes," IEEE Transactions on Information Theory, vol. 16, no. 3, pp. 359-360, May 1970.

[5] D. A. Cox, J. B. Little, and D. O'Shea, Ideals, Varieties, and Algorithms. New York: Springer, 1992.

[6] J.-C. Faugére, "A new efficient algorithm for computing Gröbner bases $\left(F_{4}\right)$, , Journal of Pure and Applied Algebra, vol. 139, no. 1-3, pp. 6188, 1999.

[7] M. Grassl, "Tables of linear codes," on-line available at http://www.codetables.de/
[8] _ " "Searching for linear codes with large minimum distance," in Discovering Mathematics with Magma - Reducing the Abstract to the Concrete, W. Bosma and J. Cannon, Eds. Heidelberg: Springer, 2006, pp. 287-313.

[9] R. Hill and P. Lizak, "Extensions of Linear Codes," in Proceedings 1995 International Symposium on Information Theory, Whistler, BC, Canada, September 17-22 1995, p. 345.

[10] R. Hill, "An Extension Theorem for Linear Codes," Designs, Codes and Cryptography, vol. 17, no. 1-3, pp. 151-157, Sept. 1999.

[11] A. Kohnert, "Update on the Extension of Good Linear Codes," Electronic Notes in Discrete Mathematics, vol. 26, pp. 81-85, Sept. 2006.

[12] _ _ " “ $(l, s)$-Extension of Linear Codes," in Proceedings Combinatorics 2006, 2007, preprint arXiv:cs/0701112 1 [cs.IT].

[13] T. Maruta, "Extendability of Linear Codes over $G F(q)$ with Minimum Distance $d, \operatorname{gcd}(d, q)=1, "$ Discrete Mathematic, vol. 266, no. 1-3, pp. 377-385, May 2003.

[14] - "A New Extension Theorem for Linear Codes," Finite Fields and Their Applications, vol. 10, pp. 674-685, 2004.

[15] — , "Extendability of Ternary Linear Codes," Designs, Codes and Cryptography, vol. 35, no. 2, pp. 175-190, May 2005.

[16] J. Simonis, “Adding a Parity-Check Bit," IEEE Transactions on Information Theory, vol. 46, no. 4, pp. 1544-1545, July 2000.

[17] N. J. A. Sloane, S. M. Reddy, and C.-L. Chen, "New Binary Codes," IEEE Transactions on Information Theory, vol. 18, no. 4, pp. 503-510, July 1972.

[18] G. White, "An Improved Minimum Weight Algorithm for Quasi-cyclic and Quasi-twisted Codes," 2005, submitted to IEEE Transactions on Information Theory.

[19] K.-H. Zimmermann, "Integral Hecke Modules, Integral Generalized Reed-Muller Codes, and Linear Codes," Technische Universität Hamburg-Harburg, Tech. Rep. 3-96, 1996. 